\title{
NEURAL GRAFTS SURVIVE POORLY IN HD
}

Neural transplants used to treat Huntington disease (HD) show disease-like degeneration in the long term, despite seeming to be successful and healthy initially. Francesca Cicchetti, Thomas Freeman and colleagues found that 10 years after transplantation, striatal grafts had degenerated extensively-more so than the host tissue. Neural transplantation as a treatment for HD will need further development before it can be considered as a viable long-term option for patients.

$\mathrm{HD}$ is caused by expansion of CAG repeats in the huntingtin (HTT) gene. This genetic defect leads to neuronal loss in the striatum. Transplantation to replace the degenerated neurons has been an exciting line of therapeutic investigation, yielding encouraging preclinical results. In rodents and primates, embryonic striatal neuron grafts have survived, become functionally integrated, and led to behavioral improvement. Furthermore, in patients with Parkinson disease, preliminary evidence exists of long-term clinical benefits from neural transplantation, with grafts surviving for 11 or 12 years.

Cicchetti and colleagues examined the brains of three patients with HD who had received striatal transplants to the caudate and putamen a decade earlier. Postmortem immunohistochemical investigation showed evidence that synaptic connections had been established, and clinical benefits had been seen in the patients at 18-month and 6-year follow-ups. The researchers found, however, that after 10 years graft survival was attenuated; all caudate grafts were lost, and the surviving putamenal grafts appeared unhealthy. The investigators conclude that despite initially promising results, neural transplantation "did not prevent the unremitting and fatal progression of HD."

In the transplanted tissue, medium spiny neurons were lost to a greater extent than interneurons, a pattern that is characteristic of HD. As the grafts were not genetically related to the host, this finding indicates that "the abnormal HD gene is not required within the striatal neurons to induce degeneration," according to Cicchetti. The mechanism by which the HTT gene defect causes cell death could, therefore, be indirect, and these results suggest a possible model for degeneration in HD.

Degeneration "may be a remote consequence of the abnormal gene expressed elsewhere in the brain," says Cicchetti. A neurotoxic environment could thereby be created, within which the transplanted neurons do not survive in the long term. Inflammatory or immune responses are thought to contribute to the hostility of this environment, as the investigators also identified microglial activity specifically targeting the neuronal components within the grafts. The findings "raise uncertainty about this potential therapeutic approach for the treatment of HD," the authors conclude, but they also indicate avenues for future development. Improving graft survival will be fundamental to developing neural transplantation as a successful treatment for HD. The investigators suggest that research should focus on reducing the effects of host-derived neurotoxicity and immune responses. Striatal grafts in patients with Parkinson disease survive longer and undergo less disease-like degeneration than those in HD, the reasons for which are not entirely clear. Clinical trials involving transplantation could also provide an important investigative tool to expose the underlying mechanisms of HD.

\section{Eleanor Beal}

Original article Cicchetti, F. et al. Neural transplants in patients with Huntington's disease undergo disease-like neuronal degeneration. Proc. Natl Acad. Sci. USA 106, 12483-12488 (2009). 